Proceedings of the 2011 Winter Simulation Conference

S. Jain, R. R. Creasey, J. Himmelspach, K. P. White, and M. Fu, eds.

\title{
AUTOMATIC SURROGATE MODEL TYPE SELECTION DURING THE OPTIMIZATION OF EXPENSIVE BLACK-BOX PROBLEMS
}

\author{
Ivo Couckuyt \\ Dirk Gorissen \\ Filip De Turck \\ Tom Dhaene
Department of Information Technology (INTEC) Computational Engineering Design Group
Ghent University - IBBT
Gaston Crommenlaan 8, Bus 201
University of Southampton
University Road \\ 9050 Ghent, BELGIUM \\ SO17 1BJ Southampton, UNITED KINGDOM
}

\begin{abstract}
The use of Surrogate Based Optimization (SBO) has become commonplace for optimizing expensive black-box simulation codes. A popular SBO method is the Efficient Global Optimization (EGO) approach. However, the performance of SBO methods critically depends on the quality of the guiding surrogate. In EGO the surrogate type is usually fixed to Kriging even though this may not be optimal for all problems. In this paper the authors propose to extend the well-known EGO method with an automatic surrogate model type selection framework that is able to dynamically select the best model type (including hybrid ensembles) depending on the data available so far. Hence, the expected improvement criterion will always be based on the best approximation available at each step of the optimization process. The approach is demonstrated on a structural optimization problem, i.e., reducing the stress on a truss-like structure. Results show that the proposed algorithm consequently finds better optimums than traditional kriging-based infill optimization.
\end{abstract}

\section{INTRODUCTION}

Surrogate Based Optimization (SBO) is an important research domain concerned with accelerating the optimization of expensive simulation problems (Eldred and Dunlavy 2006, Knowles and Nakayama 2008). In SBO intermediate surrogate models, also known as metamodels, are built to estimate the objective function in consecutive iterations.

A popular SBO approach is to use global surrogate models and emphasize on adaptive sampling, or infill criterions (Jones 2001). Starting from an initial low-fidelity surrogate model based on a limited set of samples spread over the complete design space, the infill criterion identifies new samples of interest (infill or update points) to update the surrogate model. It is crucial in global SBO to strike a correct balance between exploration - enhancing the general accuracy of the surrogate model - and exploitation - enhancing the accuracy of the surrogate model solely in the region of the (current) optimum. A well-known infill criterion that is able to effectively solve this trade-off is Expected Improvement (EI). The EI criterion has been suggested in literature as early as 1978 (Mockus, Tiesis, and Zilinskas 1978), and has been popularized by Jones et al. (Jones, Schonlau, and Welch 1998) in the Efficient Global Optimization (EGO) algorithm. An interesting discussion of the infill criteria approach is given by Jones (Jones 2001).

While the EI approach is proven to be an efficient figure of merit, the quality of the surrogate model is still arguably the most important factor in the optimization process. In the EGO algorithm the surrogate model type of choice is the kriging model as it provides the prediction variance required by EI. However other surrogate model types such as Support Vector Machines (SVM), pure Gaussian Processes (GP), Radial Basis Functions (RBF), etc. are also possible and may have superior accuracy for some problems. 


\section{Couckuyt, Gorissen, De Turck, and Dhaene}

Unfortunately it is rarely possible to choose the optimal surrogate model type upfront as the behavior of the objective function is often poorly understood or even unknown.

In this paper the authors propose to combine an Evolutionary Model Selection (EMS) algorithm with the well-known EI criterion. The EMS algorithm dynamically selects the best performing surrogate model type at each iteration of the EI algorithm. Thus, each iteration a new expensive sample point is chosen based on the EI criterion, which in its turn is based on the best surrogate model found by the EMS algorithm. This methodology is compared against traditional kriging-based infill optimization on a structural dynamics problem, namely, the optimization of a truss structure. Note that the simulation code of the truss structure is deterministic, in contrast to stochastic simulation.

Section 2 summarizes related work on automatic model selection and SBO. Subsequently, in sections 3 and 4, the EI criterion and the EMS methodology are described. Details of the application are found in section 5, while the experimental setup is described in section 6. Results and conclusion form the last two sections of this paper, i.e., 7 and 8.

\section{RELATED WORK}

Automatic model selection approaches for a single model type are quite common: (Zhang, Shao, and Li 2000, Chen, Wang, and Lee 2004, Friedrichs and Igel 2005, Lessmann, Stahlbock, and Crone 2006, Yao and $\mathrm{Xu}$ 2006, Tomioka, Nisiyama, and Enoto 2007). Integration with adaptive sampling has also been discussed (Busby, Farmer, and Iske 2007). However, these efforts do not tackle the surrogate model type selection problem, as they restrict themselves to a particular model type (e.g., SVMs or neural networks). As (Knowles and Nakayama 2008) states, "Little is known about which types of model accord best with particular features of a landscape and, in any case, very little may be known to guide this choice.". Likewise, (Solomatine and Ostfeld 2008) notes: "...it is important to stress that there are always situations when one model type cannot be applied or suffers from inadequacies and can be well complemented or replaced by another one". Thus an algorithm to solve this problem in a dynamic, fully automated way is very useful (Keys, Rees, and Greenwood 2007).

Voutchkov et. al (Voutchkov and Keane 2006) compare different surrogate model types for approximating multiple objectives during optimization. Similar work has been done by (Peter, Marcelet, Burguburu, and Pediroda 2007). A lot of work concerning the simultaneous use of multiple surrogate model types has been done in the context of evolutionary optimization (Ong, Nair, and Keane 2003, Zhou, Ong, Lim, and Lee 2007). Lim et al. (Lim, Ong, Jin, and Sendhoff 2007) benchmark different local surrogate modeling types (quadratic polynomials, GP, RBF and extreme learning machines neural networks) including the use of (fixed) ensembles, for optimization of computationally expensive simulation codes. However, these approaches still require an a priori choice of model type and does not allow any dynamic switching between the model types. Other work consists of constructing a separate surrogate model for the global search and the local search. For instance, Zhou et al. (Zhou, Ong, Nair, Keane, and Lum 2007) apply a Data Parallel Gaussian Process for the global approximation and a simple RBF model for the local search.

A different approach is taken by Lim et al. (Lim, Jin, Ong, and Sendhoff 2008) who approximate the objective function by a weighted ensemble where the weights are dynamically chosen according to the accuracy of each surrogate model, in effect adapting the surrogate model type for the problem at hand. In parallel, an independent search is applied using a simple polynomial model to account for smoothing. The work by Sanchez et. al (Sanchez, Pintos, and Queipo 2008) and Goel et. al (Goel, Haftka, Shyy, and Queipo 2007) is similar. Both provide new algorithms for generating an optimal set of ensemble members for a fixed set of data points (no sampling).

The EI approach has primarily been applied separately in conjunction with kriging models (Jones, Schonlau, and Welch 1998) and RBF models (Sóbester, Leary, and Keane 2004, Sóbester, Leary, and Keane 2005). Recently Viana et al. included other type of surrogate models (Viana and Haftka 2010). In this paper we demonstrate how the EMS algorithm described in (Gorissen, Dhaene, and DeTurck 2009) can 
Couckuyt, Gorissen, De Turck, and Dhaene

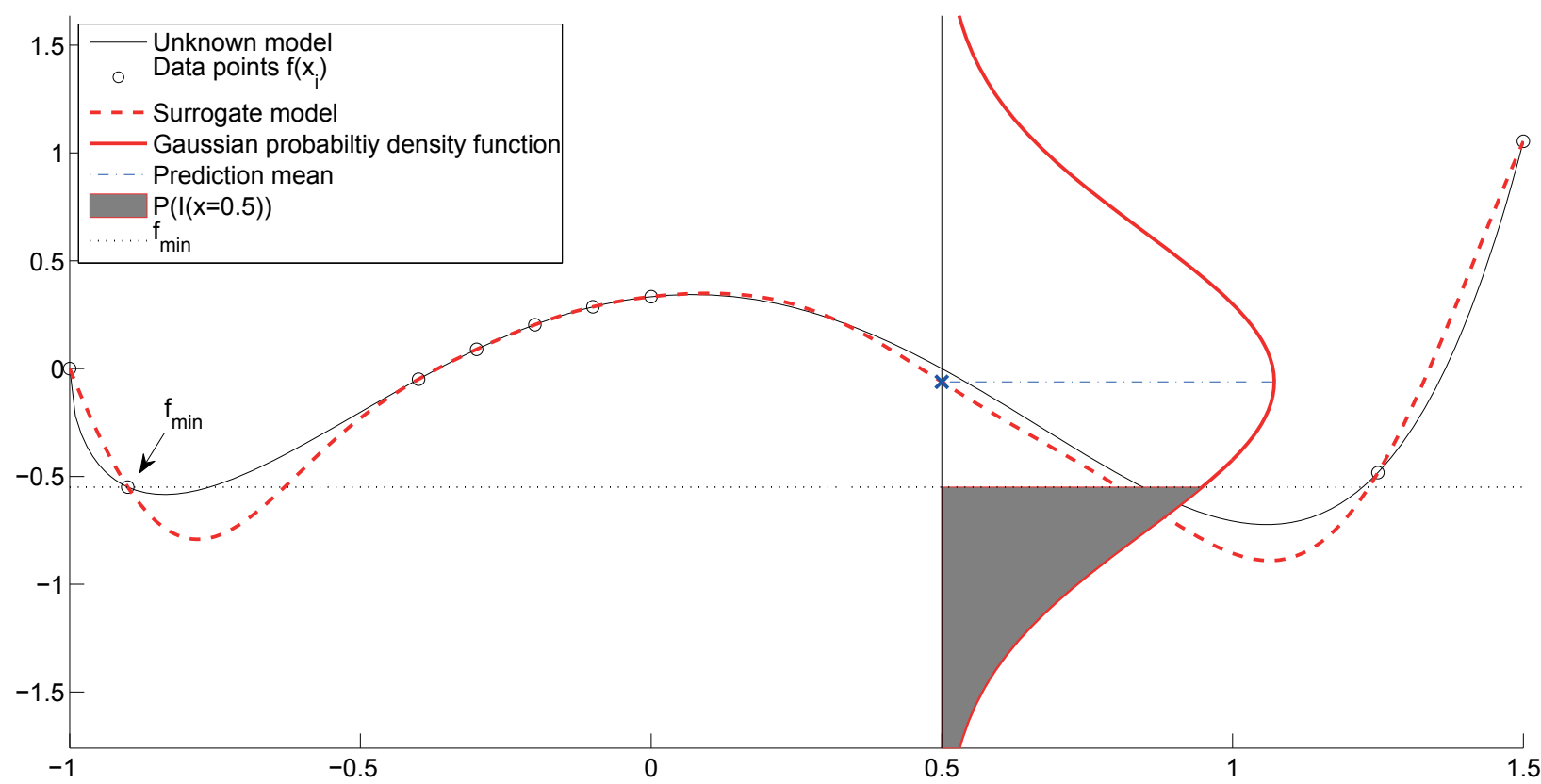

Figure 1: Graphical illustration of Gaussian Processes and E[I(x)].

be seamlessly integrated into the EI approach to allow dynamic switching of the surrogate model type and to allow the use of ensembles.

\section{EXPECTED IMPROVEMENT}

The expected improvement (EI) equation (1), defined below, can easily be interpreted graphically (see Figure 1). A kriging surrogate model, which is some form of Gaussian Processes, is fitted on a set of samples (circles). Aside from the prediction, dashed line, kriging also provides information about the uncertainty at a given point. For example, at $\mathbf{x}=0.5$ a Gaussian probability density function is drawn and expresses the uncertainty about the predicted function value. Thus, the uncertainty at any point $\mathbf{x}$ is treated as the realization of a random variable $Y(\mathbf{x})$ with mean $\hat{y}=\hat{f}(\mathbf{x})$ (=prediction) and variance $\hat{s}^{2}=\hat{\sigma}^{2}(\mathbf{x})$ (=prediction variance). Assuming the random variable is normally distributed, then the shaded area under the Gaussian probability density function is the Probability of Improvement (PoI) of any newly calculated function value $y(\mathbf{x})$ over the current minimum function value $f_{\min }$ (the dotted line), denoted as $P\left(y<f_{\min }\right)$, i.e.,

$$
\begin{aligned}
P o I=P\left(y \leq f_{\min }\right) & =\int_{-\infty}^{f_{\min }} Y(\mathbf{x}) d y=\Phi\left(\frac{f_{\text {min }}-\hat{y}}{\hat{s}}\right), \\
& =\Phi\left(\frac{f_{\text {min }}-\hat{y}}{\hat{s}}\right),
\end{aligned}
$$

where $\Phi(\cdot)$ is the standard normal cumulative distribution function. PoI is already a very useful infill criterion. However, while this criterion describes the possibility of a better minimum function value, it does not quantify how large this improvement will be.

EI quantifies the improvement and is the first moment of the shaded area, namely, every possible improvement over $f_{\min }$ multiplied by the associated likeliness. For continuous functions this is an integral defined by, 


$$
E(I)=\int_{-\infty}^{f_{\min }} I \cdot Y(\mathbf{x}) d y
$$

where

$$
I=\max \left(f_{\min }-y, 0\right) .
$$

Hence, EI can rewritten in closed form as:

$$
E(I)= \begin{cases}\left(f_{\min }-\hat{y}\right) \cdot \Phi\left(\frac{f_{\min }-\hat{y}}{\hat{s}}\right)+\hat{s} \cdot \phi\left(\frac{f_{\min }-\hat{y}}{\hat{s}}\right) & \text { if } \hat{s}>0 \\ 0 & \text { if } \hat{s}=0\end{cases}
$$

where $\phi(\cdot)$ and $\Phi(\cdot)$ denote the standard normal probability density function and standard normal cumulative distribution function, respectively.

In sum, an infill criterion is a function, also known as figure of merit, that measures how interesting a data point is in the design space. Starting from an initial low-fidelity approximation model over the design space, additional new data points (or infill points) to update the approximation model are selected by optimizing the infill criterion, see Figure $2 \mathrm{a}$.

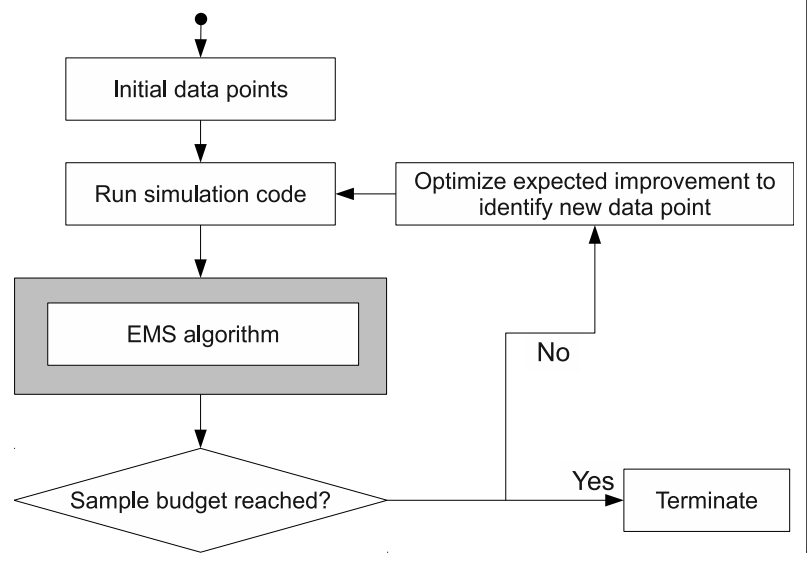

(a)

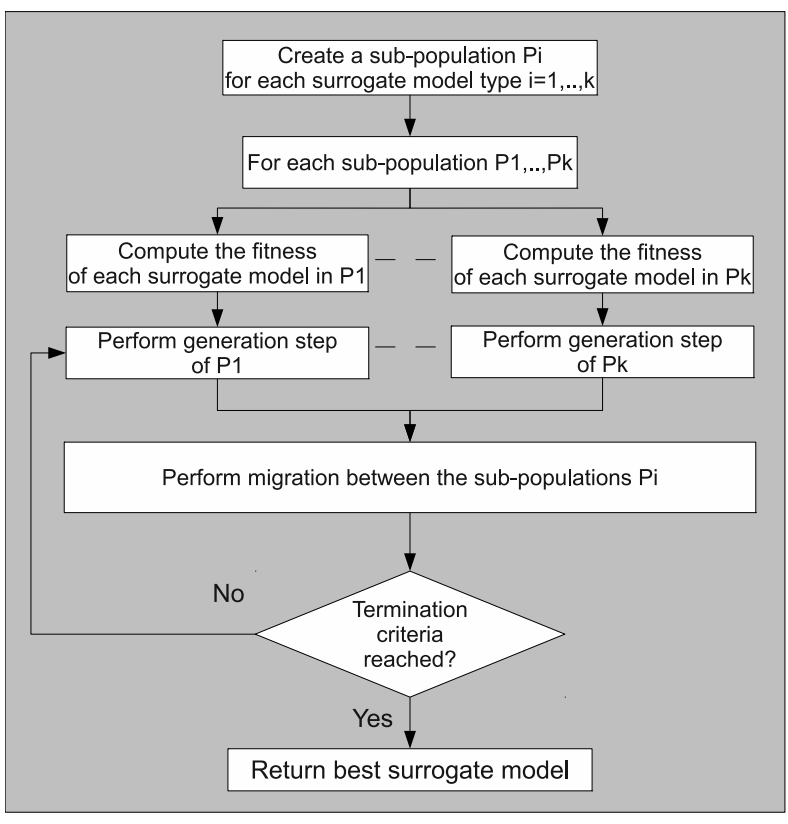

(b)

Figure 2: a) Flow chart of the expected improvement method coupled with the EMS algorithm. After creating and evaluating the initial data points the EMS algorithm identifies the surrogate model that has the best accuracy (for the given dataset). Subsequently, a new data point is selected by optimizing the expected improvement. b) Flow chart of the Evolutionary Model Selection (EMS) algorithm. For each surrogate model type under consideration a separate sub-population is created. Each sub-population creates offspring using (surrogate model type specific) genetic operators (the generation step). It is during the generation step that different surrogate model types may be combined into ensemble models. Afterwards migration occurs between the sub-populations. 
Couckuyt, Gorissen, De Turck, and Dhaene

\section{EVOLUTIONARY MODEL SELECTION}

The Evolutionary Model Selection (EMS) algorithm is based on a Genetic Algorithm (GA) with speciation (using the island model). We restrict ourselves to a brief overview of the EMS algorithm, a detailed treatment can be found in (Gorissen, Dhaene, and DeTurck 2009).

An initial sub-population (deme) is created for each surrogate model type and each deme is allowed to evolve according to an elitist GA. The different surrogate model types are implemented as Matlab objects (with full polymorphism) and each surrogate model type can choose its own representation and genetic operator implementations. This is important since it allows the genetic operators to be fully customized for each surrogate model type, allowing domain knowledge to be exploited, and improving the search efficiency. The GA is driven by a fitness function that calculates the quality of the surrogate model fit on the data. Parents are selected according to a selection algorithm (e.g., tournament selection) and offspring are generated through mutation and recombination genetic operators. The current deme population is then replaced with its offspring together with $k$ elite individuals. Once every deme has gone through a generation, migration between individuals is allowed to occur at migration interval $m_{i}$, with migration fraction $m_{f}$ and migration direction $m_{d}$ (a ring topology is used). The migration strategy is as follows: if $p$ is the population size of each deme, then the $l=\left(p * m_{f}\right)$ fittest individuals of the $i$-th deme replace the $l$ worst individuals in the next deme (defined by $m_{d}$ ). Migrants are duplicated, not removed from the source population. Thus migration ensures competition between model types since it causes model types to mix. Models which a higher fitness (e.g., better accuracy) will tend to have a higher chance of propagating to the next generation. See Figure $2 b$ for a general overview of the EMS algorithm.

An important consequence of migration is that the recombination operator (crossover) may occur on two surrogate models of different type (e.g., a rational function and a Support Vector Machine). Since a meaningful crossover between two different model types is not possible on the genotype level, the EMS implementation uses a behavioral crossover operator. The two different model types are merged together into an ensemble. Ensemble models that arise in the population through such heterogeneous crossovers, are simply treated as an additional model type (with its own operators and representation) and propagate through the population just as the other model types.

The EMS algorithm will iterate until some stopping criteria has been reached (e.g., model accuracy below $1 \%$, or maximum number of generations exceeded). In addition an extinction prevention algorithm is used to ensure no surrogate model type is driven completely extinct.

\section{PROBLEM APPLICATION}

The proposed method is applied to a structural dynamics problem. The problem is the optimal design of a two-dimensional truss, constructed by 42 Euler-Bernoulli beams. The goal is to identify a design that is optimal (or close to) with respect to passive vibration isolation. The truss structure is shown in Figure 3 and is a simplification of a truss type typically used in satellites. Furthermore, the truss simulation code is deterministic, i.e., repeated simulations return exactly the same performance (in contrast to stochastic simulation).

The beams consist each of two finite elements and are subject to a unit force excitation at node one across a $100-200 \mathrm{~Hz}$ frequency range. The two leftmost nodes are fixed (cantilevered nodes) and all the other nodes are free to move around. There are four input parameters defining the position of nodes nine and ten in the structure and one output parameter, namely, the stress that the outermost node (the tip) receives. Thus, the geometry of the structure is varied by allowing nodes nine and ten to move inside $0.9 \times 0.9$ squares while the remaining nodes are set fixed (see Figure 3). The objective is to maximize the band-averaged vibration attenuation at the tip compared to the baseline structure (= objective function). To give an idea of the complexity of the optimization problem, a 2D slice plot of the objective function is seen in Figure 5a. For an in-depth discussion of the problem the reader is referred to Keane et al. (Keane and Bright 1996). 


\section{EXPERIMENTAL SETUP}

Version 6.2.1 of the SUMO Toolbox (Gorissen, Crombecq, Couckuyt, Demeester, and Dhaene 2010) is used to optimize the truss structure and is configured as follows. The initial set of samples in the $4 D$ design space is generated by an optimal maximin Latin Hypercube Design (LHD; (Grosso, Jamali, and Locatelli 2009)) of 20 points augmented with 16 corner points, adding up to a total of 36 initial points. Several variants of the EI criteria are available in the toolbox, but for this particular application the original EI function as defined in section 3 is used to select infill points. The EI function is optimized using the DIviding RECTangles (DIRECT) algorithm of Jones et al. (Jones, Perttunen, and Stuckman 1993) to determine the next sample point to evaluate. Whenever the DIRECT algorithm is unable to obtain an unique sample a fallback criterion is optimized. This fallback criterion represents the Mean Squared Error (MSE) between the current best surrogate model and all the previous surrogate models that have been stored in the history. In effect, this identifies the locations in the domain where the prediction of surrogate models disagree the most.

The aforementioned configuration is repeated three times with different surrogate modeling strategies. The first two cases use kriging as the surrogate model type of choice. More precisely, in the first run the hyperparameters are obtained through maximum likelihood estimation (MLE) using SQPLab (Bonnans, Gilbert, Lemaréchal, and Sagastizábal 2006) (utilizing likelihood derivative information). In the second run, the hyperparameters of the kriging model are identified by Matlab's GA toolbox guided by 5 -fold cross validation. In the third run the surrogate model is produced by the EMS algorithm as described in section 4. Remark that since the EI approach is used for optimization, only model types that support a point-wise error estimation (= prediction variance) can be included in the EMS method. Thus, the surrogate model types that compete in the evolution are kriging models, RBF and Least Squares-Support Vector Machines (LS-SVMs; using (Suykens, Gestel, Brabanter, Moor, and Vandewalle 2002)). This means that, in addition to the ensemble models (that arise as a result of a crossover between two different model types), four model types will compete to fit the objective function. For this paper a straightforward weighted ensemble model is used where all weights are set equal. This was chosen since it is simple to implement and understand, and introduces no additional parameters. More complex ensemble methods such as bagging or the method used by Goel et. al. (Goel, Haftka, Shyy, and Queipo 2007), can easily be incorporated. The population size for each model type is 10 and the maximum number of generations between each sampling iteration is 15. The maximum ensemble size is set to four. The final population of the previous model type selection run is used as the initial population for the next iteration. The optimization of the EI is applied on the best performing surrogate model type as determined by the EMS algorithm and 5-fold cross validation. The error function that is minimized is the Root Relative Squared Error $(R R S E)$,
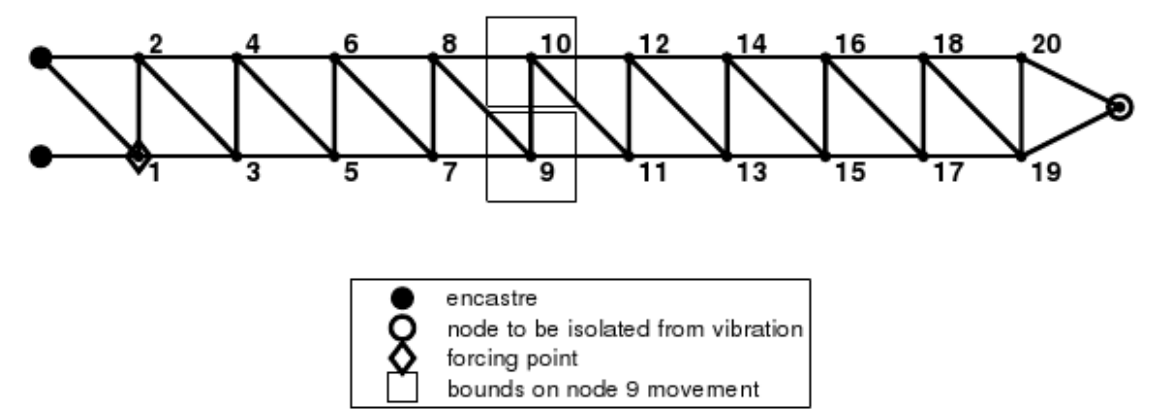

Figure 3: Truss structure consisting of 42 beams. The stress is measured at the outermost (right) node, while the two left-most nodes are fixed. 
Couckuyt, Gorissen, De Turck, and Dhaene

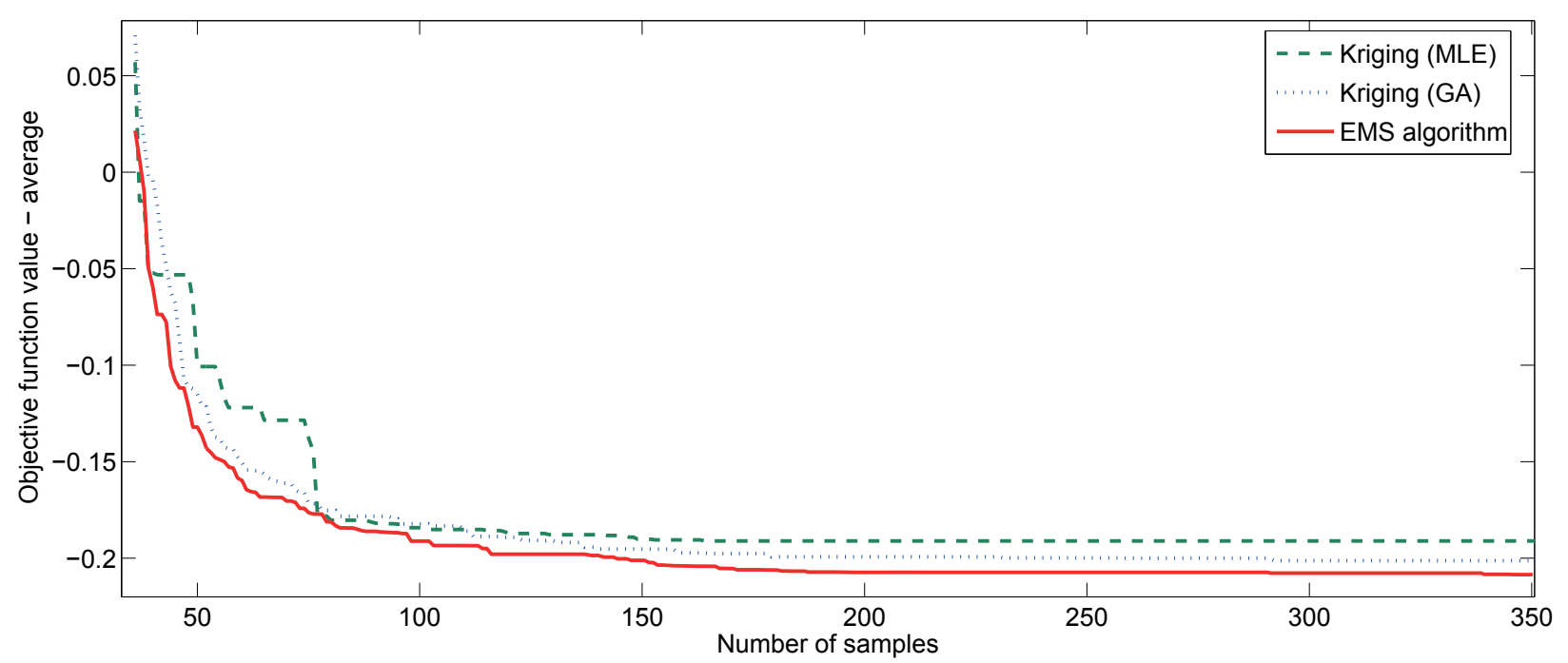

Figure 4: Evolution of the number of samples versus the minimum value.

$$
\operatorname{RRSE}(\mathbf{y}, \tilde{\mathbf{y}})=\frac{1}{n} \sqrt{\frac{\sum_{i=1}^{n}\left(y_{i}-\tilde{y}_{i}\right)^{2}}{\sum_{i=1}^{n}\left(y_{i}-\overline{\mathbf{y}}\right)^{2}}} .
$$

Each of the three different cases is allowed to run for 350 samples, in other words, the optimization process halts after 350 calls to the simulator. Finally, the tests are repeated 20 times to smooth out random effects.

\section{RESULTS}

The average minimum objective function value at each iteration of the EI approach is shown in Figure 4. It can be seen that the EMS algorithm is consistently better than the other two surrogate modeling strategies, though the difference is quite small. Worth mentioning is that the evolution of the minimum function value of the EMS algorithm is smoother than, for instance, kriging (MLE). For the latter, the function value clearly decreases in a stepwise fashion, while for the EMS algorithm other surrogate model types than kriging step in whenever they are better in approximating the intermediate set of samples, thus, a better function value is found more often.

A Box-and-whiskers plot of the final function value for the three surrogate modeling strategies is shown in Figure 5b. Kriging (GA) and the EMS algorithm are substantially more stable than using kriging (MLE), where the $\theta$ parameters are optimized using the likelihood. The EMS algorithm is also an improvement over kriging (GA) as the quantiles lie closer to the smallest function value found.

Further analysis of the EMS algorithm is rather interesting. The final best surrogate model is almost always an ensemble model, with only one case out of 20 where a sole RBF model gives the best accuracy. Furthermore, the final ensemble models consist mostly of RBF models. The share of SVM models and kriging models in those ensembles is identical.

\section{CONCLUSION}

This paper explored the use of evolutionary model selection (EMS) for surrogate based optimization (SBO). The SBO framework of EGO is coupled with the EMS framework of Gorissen et al. (Gorissen, Dhaene, and DeTurck 2009) to solve an optimization problem from structural dynamics.

It is found that for a structural dynamics problem the EMS-based method outperforms traditional EGO using just kriging models. Repeating the optimization process 20 times also shows using EMS results 


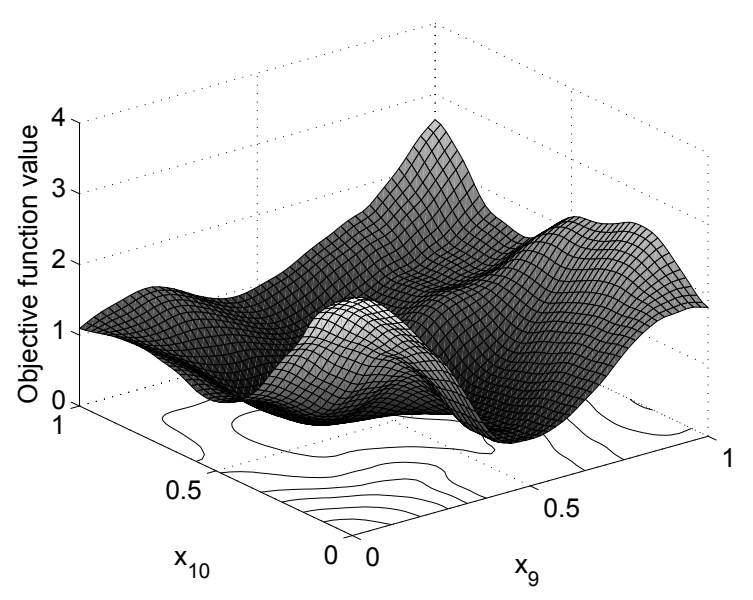

(a)

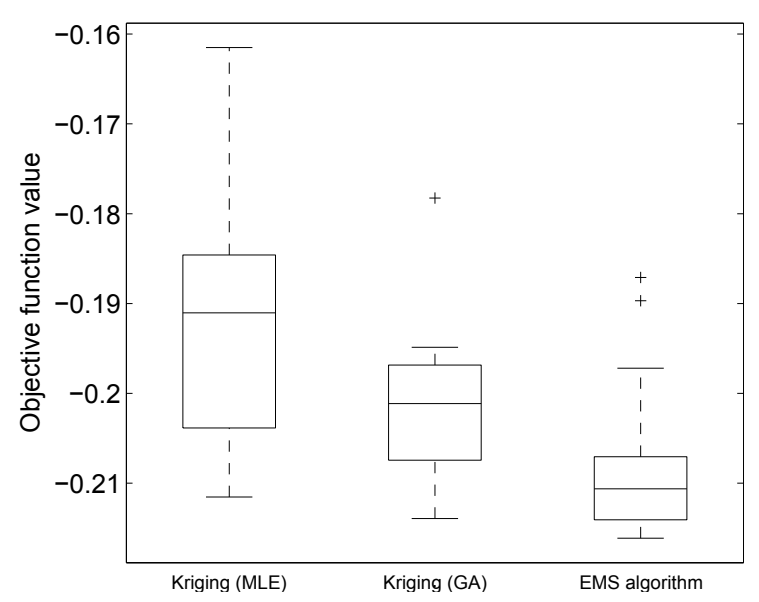

(b)

Figure 5: a) 2D slice plot of the objective function. The $y$ position of nodes 9 and 10 are set fixed to $y_{9}=0.6324$ and $y_{10}=0.0975$, respectively. b) Box-and-whiskers plot of the final optimum.

in less variance in the final optimum value than the other methods. Thus, these preliminary results are promising. Of course, using the EMS method comes at a higher computational cost as more surrogate model types are trained. The population of surrogate models of the EMS method is three times larger than kriging (GA), as three different surrogate model types compete in the population.

Further testing on a wide range of benchmark problems is currently underway to see how the performance varies on other problems. The authors are also working on expanding the range of model types that can be included in the EMS algorithm. For example by using prediction variance estimation techniques for model types that do not support the prediction variance directly.

\section{ACKNOWLEDGMENTS}

Ivo Couckuyt is funded by the Institute for the Promotion of Innovation through Science and Technology in Flanders (IWT-Vlaanderen). The authors would like to thank Alexander Forrester for kindly providing the truss simulation code.

\section{References}

Bonnans, J., J. Gilbert, C. Lemaréchal, and C. Sagastizábal. 2006. Numerical Optimization: Theoretical and Practical Aspects. Springer.

Busby, D., C. L. Farmer, and A. Iske. 2007. "Hierarchical Nonlinear Approximation for Experimental Design and Statistical Data Fitting". SIAM Journal on Scientific Computing 29 (1): 49-69.

Chen, P.-W., J.-Y. Wang, and H.-M. Lee. 2004, 25-29 July. "Model selection of SVMs using GA approach". In Neural Networks, 2004. Proceedings. 2004 IEEE International Joint Conference on, Volume 3, 2035-2040.

Eldred, M. S., and D. M. Dunlavy. 2006. "Formulations for Surrogate-Based Optimization wiht Data Fit, Multifidelity, and Reduced-Order Models". In 11th AIAA/ISSMO Multidisciplinary Analysis and Optimization Conference, Portsmouth, Virginia, AIAA-2006-7117.

Friedrichs, F., and C. Igel. 2005. "Evolutionary tuning of multiple SVM parameters.". Neurocomputing 64:107-117.

Goel, T., R. Haftka, W. Shyy, and N. Queipo. 2007. "Ensemble of surrogates”. Structural and Multidisciplinary Optimization 33:199-216. 
Gorissen, D., K. Crombecq, I. Couckuyt, P. Demeester, and T. Dhaene. 2010. "A Surrogate Modeling and Adaptive Sampling Toolbox for Computer Based Design". Journal of Machine Learning Research 11:2051-2055.

Gorissen, D., T. Dhaene, and F. DeTurck. 2009. "Evolutionary Model Type Selection for Global Surrogate Modeling". Journal of Machine Learning Research 10:2039-2078.

Grosso, A., A. Jamali, and M. Locatelli. 2009. "Finding maximin latin hypercube designs by Iterated Local Search heuristics". European Journal of Operational Research 197 (2): 541-547.

Jones, D., C. Perttunen, and B. Stuckman. 1993. "Lipschitzian optimization without the Lipschitz constant". Optimization Theory and Applications 79 (1): 157-181.

Jones, D. R. 2001. "A Taxonomy of Global Optimization Methods Based on Response Surfaces". Global Optimization 21:345-383.

Jones, D. R., M. Schonlau, and W. J. Welch. 1998. "Efficient Global Optimization of Expensive Black-Box Functions". J. of Global Optimization 13 (4): 455-492.

Keane, A. J., and A. P. Bright. 1996. "Passive vibration control via unusual geometries: experiments on model aerospace structures". Journal of Sound and Vibration 190 (4): 713-719.

Keys, A. C., L. P. Rees, and A. G. Greenwood. 2007. "Performance Measures for Selection of Metamodels to be Used in Simulation Optimization". Decision Sciences 33:31-58.

Knowles, J., and H. Nakayama. 2008. "Meta-Modeling in Multiobjective Optimization". In Multiobjective Optimization: Interactive and Evolutionary Approaches, 245-284. Berlin, Heidelberg: Springer-Verlag.

Lessmann, S., R. Stahlbock, and S. Crone. 2006, 16-21 July. "Genetic Algorithms for Support Vector Machine Model Selection". In Proceedings of the International Joint Conference on Neural Networks, 2006. IJCNN '06., 3063-3069.

Lim, D., Y. Jin, Y. S. Ong, and B. Sendhoff. 2008. "Generalizing Surrogate-assisted Evolutionary Computation". IEEE Transactions on Evolutionary Computation 14:329-355.

Lim, D., Y.-S. Ong, Y. Jin, and B. Sendhoff. 2007. "A study on metamodeling techniques, ensembles, and multi-surrogates in evolutionary computation". In Proceedings of the 9th Annual Conference on Genetic and Evolutionary Computation (GECCO 07), 1288-1295. New York, NY, USA: ACM.

Mockus, J., V. Tiesis, and A. Zilinskas. 1978. "The application of Bayesian methods for seeking the extremum". Towards Global Optimization 2:117-129.

Ong, Y.-S., P. B. Nair, and A. J. Keane. 2003. "Evolutionary Optimization of Computationally Expensive Problems via Surrogate Modeling". American Institute of Aeronautics and Astronautics Journal 41 (4): 687-696.

Peter, J., M. Marcelet, S. Burguburu, and V. Pediroda. 2007. "Comparison of surrogate models for the actual global optimization of a 2D turbomachinery flow". In Proceedings of the 7th WSEAS International Conference on Simulation, Modelling and Optimization, 46-51.

Sanchez, E., S. Pintos, and N. Queipo. 2008. "Toward an optimal ensemble of kernel-based approximations with engineering applications". Volume 36, 247-261.

Sóbester, A., S. J. Leary, and A. J. Keane. 2004. "A parallel updating scheme for approximating and optimizing high fidelity computer simulations". Structural and Multidisciplinary Optimization 27:371-383(13).

Sóbester, A., S. J. Leary, and A. J. Keane. 2005. "On the Design of Optimization Strategies Based on Global Response Surface Approximation Models". Global Optimization 33 (1): 31-59.

Solomatine, D. P., and A. Ostfeld. 2008. "Data-driven modelling : some past experiences and new approaches". Journal of hydroinformatics 10 (1): 3-22.

Suykens, J., T. V. Gestel, J. D. Brabanter, B. D. Moor, and J. Vandewalle. 2002. Least Squares Support Vector Machines. Singapore: World Scientific Publishing Co., Pte, Ltd.

Tomioka, S., S. Nisiyama, and T. Enoto. 2007, February. "Nonlinear Least Square Regression by Adaptive Domain Method With Multiple Genetic Algorithms". IEEE Transactions on Evolutionary Computation 11 (1): 1-16. 
Viana, F., and R. Haftka. 2010. "Why Not Run the Efficient Global Optimization Algorithm with Multiple Surrogates?". In 51st AIAA/ASME/ASCE/AHS/ASC Structures, Structural Dynamics, and Materials Conference, AIAA 2010-3090.

Voutchkov, I., and A. Keane. 2006, April. "Multiobjective Optimization using Surrogates". In Adaptive Computing in Design and Manufacture 2006. Proceedings of the Seventh International Conference, edited by I. Parmee, 167-175. Bristol, UK.

Yao, X., and Y. Xu. 2006. "Recent Advances in Evolutionary Computation.”. Journal of Computer Science and Technology 21 (1): 1-18.

Zhang, C., H. Shao, and Y. Li. 2000, 8-11 Oct.. "Particle swarm optimisation for evolving artificial neural network". In Systems, Man, and Cybernetics, 2000 IEEE International Conference on, Volume 4, 2487-2490.

Zhou, Z. Z., Y. S. Ong, M. H. Lim, and B. S. Lee. 2007. "Memetic Algorithm using Multi-Surrogates for Computationally Expensive Optimization Problems". Soft Computing 11 (4): 957-971.

Zhou, Z. Z., Y. S. Ong, P. B. Nair, A. J. Keane, and K. Y. Lum. 2007. "Combining Global and Local Surrogate Models to Accelerate Evolutionary Optimization”. IEEE Transactions On Systems, Man and Cybernetics - Part C 37 (1): 66-76.

\section{AUTHOR BIOGRAPHIES}

IVO COUCKUYT received his M.Sc. degree in Computer Science from the University of Antwerp (UA) in 2007. In October 2007 he joined the research group Computer Modeling and Simulation (COMS) (now merged with CoMP), supported by a research project of the Fund for Scientific Research Flanders (FWO-Vlaanderen). Starting from January 2009 he is active as a PhD student in the research group INTEC Broadband Communication Networks (IBCN) at Ghent University. At the same time he still remains affiliated with CoMP. His research activities include surrogate modeling, surrogate based optimization and inverse problems of time-consuming problems. His e-mail address is ivo.couckuyt@ugent.be

DIRK GORISSEN received a M.Sc. degree in Computer Science from the University of Antwerp in 2004 and a Masters degree in Artificial Intelligence from the Katholieke Universiteit Leuven in 2007. He continued on to the PhD level at the University of Antwerp and later at Gent University, Belgium where he obtained his PhD in Engineering Science in May 2010. During this time he also worked in research labs in Atlanta, USA and Ottawa, Canada, and he was a member of IBBT, an internationally recognized multidisciplinary ICT research center. Starting February 2010 he joined the Computational Engineering and Design Group at School of Engineering Sciences of Southampton University, UK. His research interests lie in the domain of computational engineering. Particular topics of interest include: global and local surrogate modeling for engineering design exploration and optimization, High Performance Computing, evolutionary computing, machine learning, and software engineering. His e-mail is dirk.gorissen@soton.ac.uk.

FILIP DE TURCK received his M.Sc. degree in Electronic Engineering from the Ghent University, Belgium, in June 1997. In May 2002, he obtained the Ph.D. degree in Electronic Engineering from the same university. During his Ph.D. research he was funded by the F.W.O.-V., the Fund for Scientific Research Flanders. From October 2002 until September 2008, he was a post-doctoral fellow of the F.W.O.-V. and part time professor, affiliated with the Department of Information Technology of the Ghent University. At the moment, he is a full-time professor affiliated with the Department of Information Technology of the Ghent University and the IBBT (Interdisciplinary Institute of Broadband Technology Flanders) in the area of telecommunication and software engineering. Filip De Turck is author or co-author of approximately 300 papers published in international journals or in the proceedings of international conferences. His main research interests include scalable software architectures for telecommunication network and service management, performance evaluation and design of new computational cloud services. His e-mail address 
is filip.deturck@ugent.be.

TOM DHAENE received his M.S. degree and the Ph.D. degree in Electrical Engineering from Ghent University, Ghent, Belgium, in 1989 and 1993, respectively. Since October 2000, he has been a Professor in the Computer Modeling and Simulation (COMS) research group, University of Antwerp, Antwerp, Belgium, in the Department of Mathematics and Computer Science. Starting from October 2007, he is a Professor in the INTEC Broadband Communication Networks (IBCN) research group of the Department of Information Technology (INTEC), Ghent University, Ghent, Belgium, in the Faculty of Engineering. As author or co-author, he has contributed to more than 210 peer-reviewed papers and abstracts in international conference proceedings, journals and books about computational science and engineering, numerical analysis, and computer science. He is the holder of 5 U.S. patents. His research interests include distributed scientific computing, machine learning, bio-informatics, signal integrity, electromagnetic compatibility, model order reduction, optimal design, surrogate modeling (or metamodeling) of complex systems, circuit and EM modeling of high-speed interconnections and broadband communication systems, adaptive system identification of deterministic LTI systems, and numerical analysis techniques. His e-mail address is tom.dhaene@ugent.be. 